PROCEEDINGS OF THE

AMERICAN MATHEMATICAL SOCIETY

Volume 138, Number 1, January 2010, Pages 323-332

S 0002-9939(09)10100-4

Article electronically published on September 1, 2009

\title{
A LOCAL CHARACTERIZATION OF SMOOTH PROJECTIVE PLANES
}

\author{
STEFAN IMMERVOLL, RAINER LÖWEN, AND IOACHIM PUPEZA
}

(Communicated by Ted Chinburg)

\begin{abstract}
In 2000, Bödi and Immervoll considered compact, connected smooth incidence geometries with mutually transversal point rows and mutually transversal line pencils. They made the very natural assumptions that the flag space is a $3 l$-dimensional closed smooth submanifold of the product of the point space and the line space (both of which are $2 l$-manifolds) and that both associated projections are submersions. They showed that then the number of joining lines of two distinct points and the number of intersection points of two distinct lines are constant. Here we prove that both constants are equal to one. Thus, smooth projective planes are characterized using only compactness and connectedness plus the purely local (in fact, infinitesimal) conditions stated above.
\end{abstract}

\section{INTRODUCTION}

A smooth projective plane is a triple $(P, \mathcal{L}, \mathcal{F})$, where $P$ and $\mathcal{L}$ are smooth manifolds of positive dimension, called the point space and the line space, respectively, and

$$
\mathcal{F} \subseteq P \times \mathcal{L}
$$

is a subset, called the flag space, such that the following conditions are satisfied: any two distinct points $p, q \in P$ are joined by a unique line $L=p \bigvee q \in \mathcal{L}$ (i.e., $(p, L),(q, L) \in \mathcal{F})$ and, dually, any two distinct lines $K, L \in \mathcal{L}$ meet in a unique point $p=K \bigwedge L \in P$ (i.e., $(p, K),(p, L) \in \mathcal{F})$. Moreover, the operations $\bigvee$ (join) and $\bigwedge$ (intersection) define smooth maps $\bigvee: P \times P \backslash \Delta_{P} \rightarrow \mathcal{L}$ and $\bigwedge: \mathcal{L} \times \mathcal{L} \backslash \Delta_{\mathcal{L}} \rightarrow$ $P$, respectively. Here, $\Delta_{X}$ denotes the diagonal in $X \times X$.

The classical examples are the projective planes over the real and complex numbers, over the quaternion skew field, and over the nonassociative octonion algebra; compare [22], 75.4. Projective planes are favoured objects of topological geometry; see [22]. Smooth projective planes were first studied systematically in 20], 2]. More recently, they were investigated from the point of view of symplectic geometry ([16], 17], 18]). It is quite difficult to construct explicit examples other than the classical ones (compare [21, 8]), but it has been done (20, 11]). On the other hand, the classification of smooth projective planes with large automorphism groups is possible under weaker assumptions than the corresponding task for topological projective planes (where all smoothness conditions are weakened to

Received by the editors September 14, 2008.

2000 Mathematics Subject Classification. Primary 51H25; Secondary 51H10.

Key words and phrases. Smooth projective plane.

(C)2009 American Mathematical Society Reverts to public domain 28 years from publication 
mere continuity); cf. [2], 3], 4]. The point and line manifolds of smooth projective planes are always diffeomorphic to their classical counterparts; see [17, [13].

For a smooth projective plane, it can be shown that $P$ and $\mathcal{L}$ are compact, connected manifolds of the same dimension $2 l$ and that $\mathcal{F}$ is a closed smooth $3 l$ submanifold of $P \times \mathcal{L}$. The projections

$$
\pi_{P}: \mathcal{F} \rightarrow P:(p, L) \rightarrow p
$$

and

$$
\pi_{\mathcal{L}}: \mathcal{F} \rightarrow \mathcal{L}:(p, L) \rightarrow L
$$

are submersions (i.e., all their differentials are surjective). As a consequence, the line pencils

$$
\mathcal{L}_{p}=\{L \in \mathcal{L} \mid(p, L) \in \mathcal{F}\}
$$

are $l$-dimensional submanifolds of $\mathcal{L}$. Moreover, any two distinct pencils are transversal. Dually, the point rows

$$
P_{L}=\{p \in P \mid(p, L) \in \mathcal{F}\}
$$

are $l$-dimensional submanifolds of $P$, and any two of them are transversal.

In this paper we show that the properties stated in the previous paragraph suffice to define smooth projective planes. All of these conditions, except compactness and connectednes, are of a purely local nature. It is a stunning fact that the existence and uniqueness of joining lines and of intersection points follow from these conditions.

Following [5, we therefore define a smooth local plane to be a triple $(P, \mathcal{L}, \mathcal{F})$ satisfying local conditions analogous to those stated above for smooth projective planes. To facilitate the wording of the definition, we will use a variation of the usual notion of transversality. We will say that two smooth submanifolds $X, Y$ of a smooth manifold $M$ are sharply transversal if for every point $p \in X \cap Y$ the tangent spaces satisfy the condition $\mathrm{T}_{p} M=\mathrm{T}_{p} X \oplus \mathrm{T}_{p} Y$. Note that disjoint submanifolds are sharply transversal.

Formally, we define a smooth local plane to be a triple $(P, \mathcal{L}, \mathcal{F})$, where $P$ and $\mathcal{L}$ are smooth manifolds of positive dimension and $\mathcal{F}$ is a smooth submanifold of $P \times \mathcal{L}$ such that both projections $\pi_{P}$ and $\pi_{\mathcal{L}}$, defined as above, are submersions and not injective. Moreover, we require that any two distinct lines have sharply transveral point rows and, dually, any two distinct points have sharply transversal line pencils. Here, point rows and line pencils are defined as above.

Note that there might be disjoint point rows or line pencils; also, empty line pencils or point rows may occur. Nonempty point rows $P_{L}=\pi_{P}\left(\pi_{\mathcal{L}}^{-1}(L)\right)$ and line pencils are smooth submanifolds, due to the submersion property of the projections, so that the condition of transversality is meaningful. The fibers of the submersion $\pi_{\mathcal{L}}$ are diffeomorphic to point rows and, dually, the fibers of $\pi_{P}$ are diffeomorphic to line pencils. It follows that all nonempty point rows have the same dimension $l$ and all nonempty line pencils have the same dimension $l^{\prime}$. Since $\pi_{P}$ and $\pi_{\mathcal{L}}$ are not injective, there are line pencils and point rows of cardinality $>1$. Distinct lines belonging to the same line pencil have point rows with nonempty intersection, and, dually, there are intersecting line pencils associated with distinct points. Sharp transversality of point rows and line pencils now implies that $\operatorname{dim} P=2 l$ and 
$\operatorname{dim} \mathcal{L}=2 l^{\prime}$. From the submersion property we infer the equalities $2 l+l^{\prime}=\operatorname{dim} \mathcal{F}=$ $2 l^{\prime}+l$. It follows that the dimensions are given by

$$
\operatorname{dim} P_{L}=\operatorname{dim} \mathcal{L}_{p}=l>0, \quad \operatorname{dim} P=\operatorname{dim} \mathcal{L}=2 l, \quad \operatorname{dim} \mathcal{F}=3 l
$$

here we assume that $P_{L}$ and $\mathcal{L}_{p}$ are both nonempty. Conversely, if we assume these dimension conditions, then it suffices for the definition of smooth local planes to require the ordinary transversality condition rather than sharp transversality. Also the assumption on the noninjectivity of the natural submersions $\pi_{P}$ and $\pi_{\mathcal{L}}$ is then automatically satisfied.

A smooth local plane is said to be compact or connected if both $P$ and $\mathcal{L}$ have the property in question and $\mathcal{F}$ is closed in $P \times \mathcal{L}$. In a compact and connected smooth local plane, all line pencils and point rows are nonempty and compact. This is due to the compactness of $\mathcal{F}$, the connectedness of $P$ and $\mathcal{L}$, and the fact that submersions are open maps.

Now we can formulate our main result.

Theorem 1.1. The notions of a smooth projective plane and a compact connected smooth local plane are equivalent. In other words, every compact connected smooth local plane is a smooth projective plane and vice versa.

Standard examples of smooth local planes such as the real affine plane $\left(\mathbb{R}^{2}\right.$ with the ordinary line system; the flag manifold is the graph of the incidence relation) show that noncompact smooth local planes need not be projective planes. An interesting and more complicated example is obtained from the real affine plane by forming the quotient with respect to the group of translations $(x, y) \rightarrow(x+k, y)$, $k \in \mathbb{Z}$. This geometry may be represented as a circular cylinder with its system of geodesics, equipped with its incidence graph $\mathcal{F}$. The covering map $(x, y) \rightarrow$ $\left(e^{2 \pi i x}, y\right)$ shows the equivalence of the two descriptions of this geometry. The closed geodesics of the cylinder correspond to affine lines fixed by the translation group. This implies that the space of geodesics of the cylinder is not a 2-manifold near any closed geodesic; in fact, two arbitrary closed geodesics never possess any disjoint neighborhoods in the space of geodesics. A sequence of helices cutting the closed geodesics at angles $\alpha_{\nu} \rightarrow 0$ converges to all closed geodesics simultaneously. This fact forces us to delete the closed geodesics from our geometry. The relevant submersion and transversality properties of the real affine plane carry over, and we obtain a connected smooth local plane. In this example, some pairs of lines have infinitely many common points, but there are also disjoint pairs.

Examples of disconnected smooth local planes can be obtained in many ways. One construction is to introduce additional connected components to the manifold of points, such that the new points are not incident with any line. Dually, lines may be added that do not appear in any flag. More interesting examples are obtained by taking the disjoint union of two smooth projective planes. A still more involved type of example is obtained from two smooth projective planes with the same point set $P$. One lets $\mathcal{L}$ be the disjoint union of the two line sets $\mathcal{L}_{1}$ and $\mathcal{L}_{2}$ and defines $\mathcal{F}$ in the obvious way. Again, there is a dual version of this construction. Applying our main result to the connected components of $\mathcal{F}$ and their images with respect to $\pi_{P}$ and $\pi_{\mathcal{L}}$, one sees that all compact disconnected smooth local planes can be obtained from projective planes by the repeated application of the constructions described here. 
At first sight, it seems conceivable to use a further construction principle, contradicting the preceding assertion. One could imagine having two projective planes with the same point and line spaces and disjoint flag manifolds. Then one could take the union of the flag manifolds in order to obtain a compact and connected smooth local plane. However, our main result shows that this is impossible, since we do not assume connectedness of $\mathcal{F}$, and the geometry resulting from such a construction would not be a projective plane. In fact, it follows also from known facts about the embedding of a point row in the point space of a projective plane that two lines of different planes with the same point set cannot have disjoint point rows. More precisely, the complement of a point row is always contractible, but a point row cannot be contracted within the point set; compare 22, Chap. 5 .

Examples of smooth local planes whose flag manifold is not closed in the product $P \times \mathcal{L}$ are easily obtained from projective planes by deleting some closed subset of $\mathcal{F}$, e.g. by removing a finite set of flags. In these examples, the intersection of two distinct point rows is either empty or a singleton, and the same holds for line pencils.

Our research on the comparison of projective planes and smooth local planes was triggered by the following result in 5 .

Proposition 1.2. For each compact, connected smooth local plane $\mathcal{E}$ there are positive integers $m$ and $n$ such that any two distinct points are joined by exactly $m$ lines and any two distinct lines have exactly $n$ points in common.

More precisely, this means that the line pencils of two distinct points intersect in precisely $m$ lines and, dually, the point rows of two distinct lines intersect in exactly $n$ points. The proof of Proposition 1.2 makes essential use of the assumptions of compactness and connectedness, as is to be expected in view of our examples above. The intersections in question are zero-dimensional by transversality and finite by compactness. The crucial part of the proof of Proposition 1.2 consists in showing that the cardinality of the intersection of two point rows is a locally constant function of the given pair of lines. The function is then constant by connectedness.

A similar result on the constancy of incidence numbers was proved by the first author for structures resembling smooth generalized quadrangles; see [9]. By evaluating the incidence numbers for one special instance, he used this in order to prove Thorbergson's conjecture: isoparametric hypersurfaces with four distinct principal curvatures yield generalized quadrangles; see [10].

Remember that $(m, n)=(1,1)$ is the value occuring in projective planes. Thus, in order to show that a compact, connected smooth local plane is a projective plane, it suffices to exhibit one pair of points joined by a unique line; indeed, this shows that $m=1$, and $n=1$ then follows.

Our goal in the present paper is to show that, in order to recognize smooth projective planes, it is not necessary at all to determine incidence numbers. Indeed we show that $(m, n)=(1,1)$ is the only value that can occur. This surprising fact is the essential point of our Theorem 1.1. Observe that incidence numbers contain global information, whereas we only assume local conditions such as transversality, plus compactness and connectedness. Smoothness of join and intersection is less surprising and was already proved in [5]; compare Theorems 2.1 and 2.2 below.

In concrete examples, the determination of incidence numbers can be extremely hard; see, e.g., [12. The verification of smoothness of join and intersection is easy 
for the classical examples (see [22, 75.4, where the reference should be to the first proof of 14.4 rather than 14.3), but it can be hard in other cases; compare [11. However, the main difficulty in the construction of concrete examples of smooth projective planes is not the verification of the smoothnes properties itself (e.g., by means of transversality conditions), but to find appropriate candidates for which the verification of these properties is feasible. In fact, many well-understood examples of compact, connected projective planes do not admit the structure of a smooth projective plane. There are, e.g., no smooth projective translation planes except for the classical ones (see 21]), whereas it is quite easy to construct examples of nonclassical smooth affine translation planes. All known evidence indicates that smoothness is a rare property in compact projective planes.

The present work grew out of the third author's Diploma thesis [19], in which the two-dimensional case was investigated.

\section{General Results}

We remark that the notions of a smooth projective plane and a smooth local plane are both of a self-dual nature. This means that the structure obtained by interchanging $P$ and $\mathcal{L}$ satisfies the same axioms. Hence, every general result about these structures remains true if we swap the roles of points and lines.

Throughout this section, $\mathcal{E}=(P, \mathcal{L}, \mathcal{F})$ denotes a smooth local plane. The following is one of the main results of [5], and it will play a crucial role in our proof.

Theorem 2.1. Let $\mathcal{E}=(P, \mathcal{L}, \mathcal{F})$ be a smooth local plane and consider a pair of distinct lines $L_{1}, L_{2} \in \mathcal{L}$ intersecting in $p \in P$. There are open neighborhoods $\mathcal{U}_{i}$ of $L_{i}$ in $\mathcal{L}$ with $i=1,2$, and $V$ of $p$ in $P$, such that any two distinct lines $K_{1} \in \mathcal{U}_{1}$ and $K_{2} \in \mathcal{U}_{2}$ intersect in exactly one point in $V$ (however, there may be additional intersection points outside $V$ ). Moreover, the intersection map restricted to $\mathcal{U}_{1} \times \mathcal{U}_{2}$ and $V$, i.e., the map that assigns to a pair of lines in $\mathcal{U}_{1} \times \mathcal{U}_{2}$ their intersection point in $V$, is smooth.

It is worthwhile to state explicitly the dual of this result:

Theorem 2.2. Let $\mathcal{E}=(P, \mathcal{L}, \mathcal{F})$ be a smooth local plane and consider a pair of distinct points $p_{1}, p_{2} \in P$, joined by $L \in \mathcal{L}$. There are open neighborhoods $V_{i}$ of $p_{i}$ in $P$ with $i=1,2$, and $\mathcal{U}$ of $L$ in $\mathcal{L}$, such that any two distinct points $q_{1} \in V_{1}$ and $q_{2} \in V_{2}$ are joined by exactly one line in $\mathcal{U}$. Moreover, the join map restricted to $V_{1} \times V_{2}$ and $\mathcal{U}$, i.e. the map that assigns to a pair of points in $V_{1} \times V_{2}$ their joining line in $\mathcal{U}$, is smooth.

Essentially by combining these two results, we obtain the following proposition, which in the case $m, n \geq 2$ allows us to introduce a kind of local coordinate system for the point set. We shall need to restrict the domain of an intersection map $\bigwedge_{V}$ as in Theorem 2.1 to a suitable product of pencils or, more precisely, to a set of type $\left(\mathcal{U}_{1} \times \mathcal{U}_{2}\right) \cap\left(\mathcal{L}_{q} \times \mathcal{L}_{r}\right)$. In this case $\bigwedge_{V}$ retains its smoothness property, since $\mathcal{L}_{p}$ is a smoothly embedded submanifold of $\mathcal{L}$. The same remark holds for the restriction of the domain of the join map to $\{p\} \times V_{2}$ with $p \in V_{1}$.

Proposition 2.3. Let $p \in P$ be a fixed point, $L_{1}, L_{2} \in \mathcal{L}_{p}$ be two distinct lines and $q \in \bigwedge\left(L_{1}, L_{2}\right) \backslash\{p\}$. Then, there exist open neighborhoods $\mathcal{U}_{i}$ of $L_{i}$ in $\mathcal{L}_{p}$ with $i=1,2$, and $V$ of $q$ in $P$, such that $\bigwedge_{V}: \mathcal{U}_{1} \times \mathcal{U}_{2} \rightarrow V$ is a diffeomorphism with the inverse map given by $q \rightarrow \bigwedge_{V}^{-1}(q)=\left(\bigvee_{\mathcal{U}_{1}}(p, q), \bigvee_{\mathcal{U}_{2}}(p, q)\right)$. 
Proof. 1. Applying Theorem 2.2 twice, namely for both joining lines $L_{1}$ and $L_{2}$ of $p, q \in P$, yields that there exists an open neighborhood $V$ in $P$ of $q$ and open neighborhoods $\mathcal{U}_{i}^{\prime}$ in $\mathcal{L}$ of $L_{i}$ such that for every $q^{\prime} \in V$, the points $q^{\prime}$ and $p$ are joined by exactly one line in $\mathcal{U}_{i}^{\prime}$ for $i=1,2$, respectively. The sets $\mathcal{U}_{i}:=\mathcal{U}_{i}^{\prime} \cap \mathcal{L}_{p}$ with $i=1,2$ are open neighborhoods of $L_{i}$ in $\mathcal{L}_{p}$. As discussed in the preceding remark, the map $\left(\bigvee_{\mathcal{U}_{1}}(p,),. \bigvee_{\mathcal{U}_{2}}(p,).\right): V \rightarrow \mathcal{U}_{1} \times \mathcal{U}_{2}$ is smooth.

2. Conversely, Theorem 2.1 guarantees the existence of two open neighborhoods of $L_{1}$ and $L_{2}$, respectively, in $\mathcal{L}$ and of an open neighborhood $V^{\prime}$ in $P$ of $q$, such that each pair of lines chosen from the neighborhoods intersects exactly once in $V^{\prime}$, and $\Lambda_{V^{\prime}}$ is smooth. By intersecting each of the two neighborhoods of the lines with $\mathcal{L}_{p}$, we obtain two open neighborhoods of $L_{1}$ and $L_{2}$ in $\mathcal{L}_{p}$, and $\bigwedge_{V^{\prime}}$ restricted to these neighborhoods is smooth. Now we can assume without loss of generality that the two open neighborhoods of $L_{1}$ and $L_{2}$ in $\mathcal{L}_{p}$ are exactly $\mathcal{U}_{1}$ and $\mathcal{U}_{2}$ from the first part of the proof and, in conclusion, that the sets $V$ and $V^{\prime}$ coincide. For every point $q^{\prime} \in V$ the equality $\bigwedge_{V}^{-1}\left(q^{\prime}\right)=\left(\bigvee_{\mathcal{U}_{1}}\left(p, q^{\prime}\right), \bigvee_{\mathcal{U}_{2}}\left(p, q^{\prime}\right)\right)$ holds.

Our next aim is to identify the topological nature of line pencils and point rows. This will be achieved by comparison with a suitable Graßmann manifold. As usual, the set of all $l$-dimensional subspaces of a real vector space $V$ of finite dimension $d$ is denoted by $\mathrm{G}_{l} V$. This set becomes a manifold of dimension $l(d-l)$, known as a Graßmann manifold, if it is endowed with a suitable topology; see [1, VI, $\S 3.5$ or 22], 64.3. A sequence $U_{\nu}$ of subspaces converges to a subspace $U$ with respect to this topology if, and only if, the following condition is satisfied: for every convergent sequence of vectors $u_{\nu} \in U_{\nu}$, the limit $u$ belongs to $U$. To verify this, choose an orthonormal basis of $U_{\nu}$ for each $\nu$ and use the compactness of the space of orthonormal systems $e_{1}, \ldots, e_{l} \in V$.

Lemma 2.4. Let $p \in P$ be a point with $\mathcal{L}_{p} \neq \emptyset$. The map $\mathrm{T}_{p}: \mathcal{L}_{p} \rightarrow \mathrm{G}_{l} \mathrm{~T}_{p} P$ which sends a line $L \in \mathcal{L}_{p}$ to its tangent space $\mathrm{T}_{p} P_{L}$ is continuous and injective.

Proof. Injectivity is immediate from the condition that the point rows of two distinct lines are transversal. In order to prove the continuity of $\mathrm{T}_{p}$, we will give a description of $\mathrm{T}_{p} P_{L}$ which makes this more or less obvious.

For $L \in \mathcal{L}_{p}$, consider the fiber $\pi_{\mathcal{L}}^{-1}(L)=\left\{(q, L) \mid q \in P_{L}\right\} \subseteq \mathcal{F}$. The restriction of $\pi_{P}$ maps this fiber diffeomorphically onto the point row $P_{L}$, while $\pi_{\mathcal{L}}$ is constant on $\pi_{\mathcal{L}}^{-1}(L)$. Therefore, the tangent space $\mathrm{T}_{(p, L)}\left(\pi_{\mathcal{L}}^{-1}(L)\right)$ is $l$-dimensional and is contained in the kernel of $\mathrm{T}_{(p, L)} \pi_{\mathcal{L}}$, which is also $l$-dimensional (remember that $\pi_{\mathcal{L}}$ is a submersion). Thus, we have

$$
\mathrm{T}_{(p, L)}\left(\pi_{\mathcal{L}}^{-1}(L)\right)=\operatorname{ker} \mathrm{T}_{(p, L)} \pi_{\mathcal{L}}
$$

and

$$
\mathrm{T}_{(p, L)} \pi_{P}\left(\mathrm{~T}_{(p, L)}\left(\pi_{\mathcal{L}}^{-1}(L)\right)\right)=\mathrm{T}_{p} P_{L}
$$

This is the description we desired.

Now let $L_{\nu} \rightarrow L$ be a convergent sequence in $\mathcal{L}_{p}$. According to the remarks preceding the statement of this lemma, we have to show that for every convergent sequence $u_{\nu} \rightarrow u$ in $\mathrm{T}_{p} P$ with $u_{\nu} \in \mathrm{T}_{p} P_{L_{\nu}}$, the limit $u$ belongs to $\mathrm{T}_{p} P_{L}$.

For each $\nu$, consider the unique vector $v_{\nu} \in \mathrm{T}_{\left(p, L_{\nu}\right)}\left(\pi_{\mathcal{L}}^{-1}\left(L_{\nu}\right)\right)=\operatorname{ker} \mathrm{T}_{\left(p, L_{\nu}\right)} \pi_{\mathcal{L}}$ which is sent to $u_{\nu}$ by $\mathrm{T}_{(p, L)} \pi_{P}$. With respect to the product structure given by $\mathrm{T}_{\left(p, L_{\nu}\right)} \mathcal{F} \subseteq \mathrm{T}_{p} P \times \mathrm{T}_{L_{\nu}} \mathcal{L}$, we have $v_{\nu}=\left(u_{\nu}, 0\right)$. Therefore, $v_{\nu}$ converges to $(u, 0)$, 
and we see that $(u, 0) \in \operatorname{ker} \mathrm{T}_{(p, L)} \pi_{\mathcal{L}}=\mathrm{T}_{(p, L)}\left(\pi_{\mathcal{L}}^{-1}(L)\right)$. Applying $\mathrm{T}_{(p, L)} \pi_{P}$, we obtain that $u \in \mathrm{T}_{p} P_{L}$.

Proposition 2.5. Let $\mathcal{E}=(P, \mathcal{L}, \mathcal{F})$ be a compact, connected smooth local plane. Then every line pencil $\mathcal{L}_{p}, p \in P$, and every point row $P_{L}, L \in \mathcal{L}$, is homeomorphic to the l-dimensional sphere $\mathbb{S}_{l}$.

Proof. It suffices to prove one of these dual statements; we choose the first one. First recall that $\mathcal{L}_{p}$ is compact and nonempty, since we are dealing with a compact connected smooth local plane. According to Lemma 2.4, it suffices therefore to show that the set $\mathcal{T}_{p}$ of all tangent spaces $\mathrm{T}_{p} P_{L}, L \in \mathcal{L}_{p}$, is homeomorphic to $\mathbb{S}_{l}$. Now these tangent spaces are mutually complementary $l$-dimensional vector subspaces of the $2 l$-dimensional space $\mathrm{T}_{p} P$. This means that $\mathcal{T}_{p}$ is a so-called partial spread. Furthermore, $\mathcal{T}_{p} \approx \mathcal{L}_{p}$ is a compact $l$-manifold. It is known that a partial spread which is a compact $l$-manifold is a spread; i.e., it covers the whole space (see [14, 1.7 ), and then it is homeomorphic to the $l$-sphere (see [22], 64.8).

\section{TWO-Dimensional Planes}

This section contains the proof of Theorem 1.1 in the case $l=1$. Since the conditions $n=1$ and $m=1$ are equivalent, a hypothetical counterexample $(P, \mathcal{L}, \mathcal{F})$ to Theorem 1.1 satisfies both $n \geq 2$ and $m \geq 2$. We will investigate this counterexample until a contradiction becomes apparent.

1. Choose a flag $(p, K)$ and a sequence of points $p_{\nu} \in P_{K}$ distinct from $p$ and converging to $p$. For each $\nu$, choose a line $K_{\nu}$ distinct from $K$ and containing the points $p$ and $p_{\nu}$. We claim that $K_{\nu} \rightarrow K$. Assume that this is not the case. As the compact pencil $\mathcal{L}_{p}$ contains the sequence $\left(K_{\nu}\right)$, we could then pass to a subsequence for which $\left(K_{\nu}\right)$ converges to a line $K^{\prime} \neq K$ in $\mathcal{L}_{p}$. But this would contradict Theorem 2.1? by construction of the sequence, every neighborhood of $p$ contains two intersection points of $K_{\nu}$ and $K$ for large $\nu$, whereas Theorem 2.1 asserts that there is only one.

2. Choose a line $L \in \mathcal{L}_{p}$ distinct from $K$; then $K$ and $L$ intersect in a second point $q \neq p$, as shown in Figure 1. According to Proposition 2.3 there are neighborhoods $\mathcal{U}, \mathcal{V} \subseteq \mathcal{L}_{q}$ of $L$ and $K$, respectively, and a neighborhood $W$ of $p$ in $P$ such that $\bigwedge_{W}$ is a diffeomorphism $\mathcal{U} \times \mathcal{V} \rightarrow W$. We may assume that both $\mathcal{U}$ and $\mathcal{V}$ are diffeomorphic to the interval $[-1,1]$; hence we have constructed a differentiable coordinate system $\varphi: W \rightarrow[-1,1]^{2}$. Write $\varphi(w)=(x, y)$ and note that the horizontal and vertical fibers defined by $y=y_{0}$ and $x=x_{0}$ for $x_{0}, y_{0} \in[-1,1]$ are linear sets, i.e., each fiber is contained in the point row of a line. Moreover, the point row $P_{K}$ contains one of the horizontal fibers, say the fiber $y=0$.

3. We investigate the intersection of $P_{K_{\nu}}$ with the boundary of $W$. The fact that $\mathcal{F}$ is closed in $P \times \mathcal{L}$ implies that the fibers $y= \pm 1$ are disjoint from the point rows $P_{K_{\nu}}$ for large $\nu$; indeed, every accumulation point of any sequence $w_{\nu} \in P_{K_{\nu}}$ must belong to $P_{K}$. For the same reason, any sequence of intersection points of $P_{K_{\nu}}$ with a fiber $x= \pm 1$ converges to the point $\varphi^{-1}( \pm 1,0)$ on $P_{K}$. By an argument similar to step 1 of this proof, it follows that for large $\nu$, there is at most one intersection point of $P_{K_{\nu}}$ with each of these vertical fibers.

4. The point $q$ considered in step 2 is contained in $P_{K}$; hence $K_{\nu} \rightarrow K$ implies that there is a sequence $q_{\nu} \rightarrow q$ such that $q_{\nu} \in P_{K_{\nu}}$. One may apply Proposition 2.3 in order to see this. The pair of points $p_{\nu}, p$ splits the point row $P_{K_{\nu}} \approx \mathbb{S}_{1}$ into two 


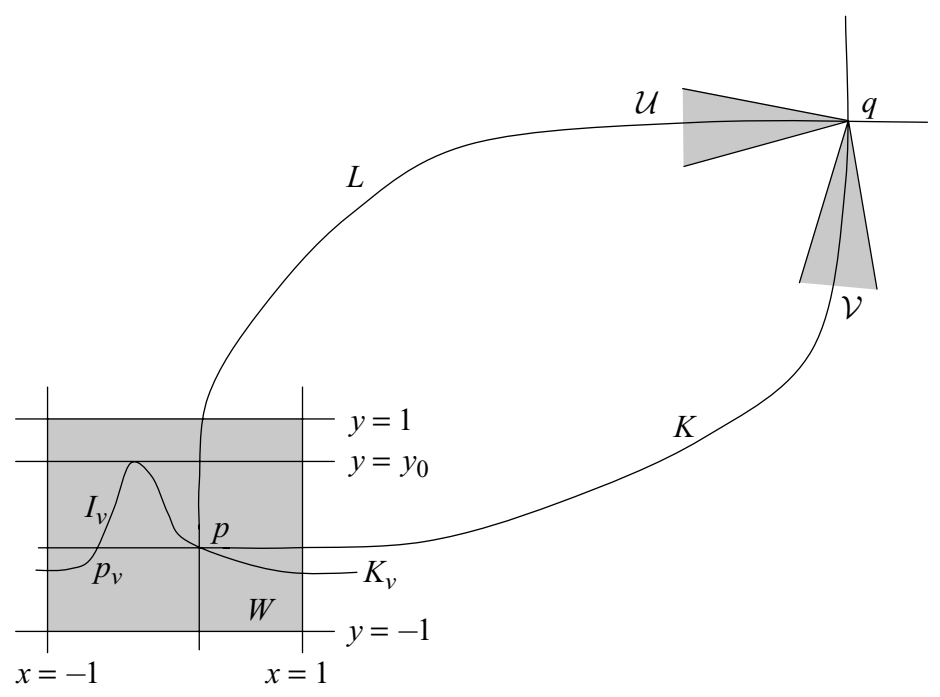

Figure 1

closed intervals $I_{\nu}$ and $J_{\nu}$. One of them contains the point $q_{\nu}$ and, hence, intersects both of the vertical fibers $x= \pm 1$. We may assume that this is the interval $J_{\nu}$. It follows from step 3 that the other interval $I_{\nu}$ is contained in the interior of $W$.

5. We are now ready to diagnose a contradiction. An interval $I_{\nu} \subseteq P_{K_{\nu}}$ contained in the interior of $W$ has end points satisfying $y=0$ and contains a point $\varphi^{-1}\left(x_{0}, y_{0}\right)$ with maximal absolute value $\left|y_{0}\right|$. Then $y_{0} \neq 0$ holds because $K$ and $K_{\nu}$ intersect in only finitely many points. We see that the point row of $K_{\nu}$ touches the point row containing the fiber $y=y_{0}$ without crossing it, contradicting the fact that point rows are transversal. This ends the proof of Theorem 1.1 in the case $l=1$.

\section{HigheR-DIMENSIONAL PLANES}

In this section, we show that the parameter $m$ of a compact connected smooth local plane with $l>1$ cannot take values other than $m=1$. By duality, or simply because $m=1$ implies $n=1$, this will complete the proof of Theorem 1.1 .

1. Choose a point $p \in P$. We consider the subset

$$
\mathcal{F}_{p}:=\pi_{\mathcal{L}}^{-1}\left(\mathcal{L}_{p}\right)=\left\{(q, L) \in \mathcal{F} \mid L \in \mathcal{L}_{p}\right\} .
$$

Since $\mathcal{L}_{p}$ is a smooth $l$-dimensional submanifold of the $2 l$-manifold $\mathcal{L}$ and $\pi_{\mathcal{L}}: \mathcal{F} \rightarrow$ $\mathcal{L}$ is a submersion, we conclude that $\mathcal{F}_{p}$ is a smooth $2 l$-dimensional submanifold of the $3 l$-manifold $\mathcal{F}$. By construction, the restriction $\pi_{\mathcal{L}}: \mathcal{F}_{p} \rightarrow \mathcal{L}_{p}$ is still a submersion. Since $\mathcal{F}_{p}$ is compact, a theorem of Ehresmann implies that this restricted map is a locally trivial fibration; see [6], 8.12. According to Proposition 2.5] both the base and the fibers of this fibration are homeomorphic to the sphere $\mathbb{S}_{l}$. The fibration $\mathcal{F}_{p} \rightarrow \mathcal{L}_{p}$ has a continuous section $L \rightarrow(p, L)$. Removing the image of this section from the total space $\mathcal{F}_{p}$, we obtain the open subset

$$
\mathcal{F}_{p}^{\prime}:=\mathcal{F}_{p} \backslash\left(\{p\} \times \mathcal{L}_{p}\right) .
$$

It is easy to see that the restriction $\pi_{\mathcal{L}}: \mathcal{F}_{p}^{\prime} \rightarrow \mathcal{L}_{p}$ is again a locally trivial fibration, with fibers homeomorphic to $\mathbb{R}^{l}$. Now the exact homotopy sequence of the fibration 
obtained by restriction,

$$
\cdots \rightarrow \pi_{k} \mathbb{R}^{l} \rightarrow \pi_{k} \mathcal{F}_{p}^{\prime} \rightarrow \pi_{k} \mathbb{S}_{l} \rightarrow \pi_{k-1} \mathbb{R}^{l} \rightarrow \ldots,
$$

shows that $\pi_{\mathcal{L}}$ induces isomorphisms between the homotopy groups of $\mathcal{F}_{p}^{\prime}$ and of $\mathbb{S}_{l}$ in all dimensions. By a theorem of Whitehead (see [23, Theorem 7.5.9) the map $\pi_{\mathcal{L}}$ induces isomorphisms of homology groups in all dimensions as well. So we have reached the conclusion that

$$
H_{k} \mathcal{F}_{p}^{\prime} \cong H_{k} \mathbb{S}_{l}
$$

holds for all $k$. Moreover, $\mathcal{F}_{p}^{\prime}$ is (connected and) simply connected, because we are assuming that $l>1$.

2. Now we consider the map

$$
\pi_{P}: \mathcal{F}_{p}^{\prime} \rightarrow P \backslash\{p\}
$$

Every point $q \neq p$ is joined to $p$ by exactly $m$ lines, and at each of these lines we can use Theorem 2.2 in order to construct a local inverse map. This implies that the map $\pi_{P}$, restricted as above, is an $m$-fold covering map. (Being a proper submersion, this map also satisfies the hypotheses of Ehresmann's theorem quoted above, which yields an alternative argument for the covering property.) Since $\mathcal{F}_{p}^{\prime}$ is simply connected by step 1 , it follows that this is a universal covering and the fundamental group $\pi_{1}(P \backslash\{p\})$ has order $m$.

The entire space $P$ may be described as the union of a $2 l$-dimensional ball with $P \backslash\{p\}$, and the intersection of these two sets is a punctured $2 l$-ball, hence homotopy equivalent to $\mathbb{S}_{2 l-1}$. Again since we assume that $l>1$, the punctured ball is simply connected, and we conclude that $\pi_{1} P$ is isomorphic to $\pi_{1}(P \backslash\{p\})$ by van Kampen's theorem (see, e.g., [15, Chap. IV).

Therefore, there is an $m$-sheeted universal covering $\rho: \tilde{P} \rightarrow P$. Deleting the $m$ inverse images of $p$ from $\tilde{P}$, we obtain an $m$-fold covering $\rho: \tilde{P}^{\prime} \rightarrow P \backslash\{p\}$ by restriction. The number of sheets equals the order of the fundamental group of $P \backslash\{p\}$; thus we have constructed another universal covering space for $P \backslash\{p\}$. (That $\tilde{P}^{\prime}$ is simply connected follows also from $\pi_{1} \tilde{P}=1$ by the van Kampen argument used before.) Thus, $\mathcal{F}_{p}^{\prime}$ and $\tilde{P}^{\prime}$ are homeomorphic, and we may obtain $\tilde{P}$ from $\mathcal{F}_{p}^{\prime}$, up to homeomorphism, by adding $m$ points.

3. The top homology $H_{2 l} \tilde{P}$ of the simply connected (hence orientable) compact $2 l$-manifold $\tilde{P}$ is isomorphic to $\mathbb{Z}$; see [7, 22.28. On the other hand, we may describe $\tilde{P}$ as the union of $\mathcal{F}_{p}^{\prime}$ with $m$ compact discs $D_{i} \approx \mathbb{D}_{2 l}, i=1, \ldots, m$, such that these discs intersect $\mathcal{F}_{p}^{\prime}$ in $m$ punctured discs $D_{i} \backslash\left\{p_{i}\right\}$. Evaluating the Mayer-Vietoris sequence of this union $([7,17.6)$ using the result of step 1 , we obtain an exact sequence

$$
0 \rightarrow H_{2 l} \tilde{P} \rightarrow \bigoplus_{i=1}^{m} H_{2 l-1}\left(D_{i} \backslash\left\{p_{i}\right\}\right) \rightarrow 0,
$$

and this is only possible if $m=1$. This ends the proof of Theorem 1.1

\section{REFERENCES}

[1] N. Bourbaki, General Topology, Part 2. Hermann, Paris, 1966. MR0205211 (34:5044b)

[2] R. Bödi, Smooth stable and projective planes, Habilitation Thesis, University of Tübingen, 1996.

[3] R. Bödi, 16-dimensional smooth projective planes with large collineation groups, Geom. Dedic. 72, pp. 283-298, 1998. MR1647708 (99m:51025) 
[4] R. Bödi, Smooth Hughes planes are classical, Arch. Math. 73, pp. 73-80, 1999. MR:1696544 (2000i:51038)

[5] R. Bödi, S. Immervoll, Implicit characterizations of smooth incidence geometries, Geom. Dedic. 83, pp. 63-76, 2000. MR.1800011 (2001j:51018)

[6] T. Bröcker, K. Jänich, Introduction to Differential Topology, Cambridge University Press, Cambridge, 1987. MR674117(83i:58001)

[7] M. Greenberg, Lectures on Algebraic Topology, Benjamin, New York-Amsterdam, 1967. MR0215295 (35:6137)

[8] S. Immervoll, Glatte affine Ebenen mit großer Automorphismengruppe und ihr projektiver Abschluß, Diploma Thesis, University of Tübingen, 1998.

[9] S. Immervoll, Smooth generalized quadrangles and isoparametric hypersurfaces of Clifford type, Forum Math. 14, pp. 877-899, 2002. MR.1932524 (2003i:53083)

[10] S. Immervoll, Isoparametric hypersurfaces and smooth generalized quadrangles, J. Reine Angew. Math. 554, pp. 1-17, 2003. MR.1952166 (2003m:51018)

[11] S. Immervoll, Real analytic projective planes with large automorphism groups, Adv. Geom. 3, pp. 163-176, 2003. MR1967997 (2004b:51013)

[12] N. Knarr, R. Löwen, Four-dimensional compact projective planes admitting an affine Hughes group, Result. Math. 38, pp. 270-306, 2000. MR 1799719 (2001h:51022)

[13] L. Kramer, S. Stolz, A diffeomorphism classification of manifolds which are like projective planes, J. Differ. Geom. 77, pp. 177-188, 2007. MR2355782 (2008g:57032)

[14] R. Löwen, Topological pseudo-ovals, elation Laguerre planes, and elation generalized quadrangles, Math. Z. 216, pp. 347-369, 1994. MR1283074 (95h:51025)

[15] W.S. Massey, Algebraic Topology: An Introduction, Graduate Texts in Math., vol. 56, Springer-Verlag, New York-Heidelberg, 5th printing, 1977. MR0448331 (56:6638)

[16] B. McKay, Dual curves and pseudoholomorphic curves, Selecta Math. 9, pp. 251-311, 2003. MR.1993485 (2004g:53099)

[17] B. McKay, Smooth projective planes, Geom. Dedic. 116, pp. 157-202, 2005. MR 2195446 (2007g:53085)

[18] B. McKay, Almost complex rigidity of the complex projective plane, Proc. Amer. Math. Soc. 135, pp. 597-603, 2007. MR 2255307 (2007f:53119)

[19] I. Pupeza, A local characterization of two-dimensional smooth projective planes, Diploma Thesis, Technische Universität Braunschweig, 2007.

[20] J. Otte, Differenzierbare Ebenen, Dissertation, Universität Kiel, 1992.

[21] J. Otte, Smooth projective translation planes, Geom. Dedic. 58, pp. 203-212, 1995. MR1358234 (96h:51011)

[22] H. Salzmann, D. Betten, T. Grundhöfer, H. Hähl, R. Löwen, M. Stroppel, Compact Projective Planes, de Gruyter, Berlin, 1995. MR1384300 (97b:51009)

[23] E.H. Spanier, Algebraic Topology, McGraw-Hill, New York, 1966. MR0210112 (35:1007)

Mathematisches Institut, Universität Tübingen, Auf der Morgenstelle 10, D-72076 TüBIngen, Germany

E-mail address: stim@fa.uni-tuebingen.de

Institut für Analysis und Algebra, Technische Universität Braunschweig, PockelsStrasse 14, D-38106 Braunschweig, Germany

E-mail address: r.loewen@tu-bs.de

Institut für Analysis und Algebra, Technische Universität Braunschweig, PockelsStrasse 14, D-38106 Braunschweig, Germany

Current address: Max-Planck-Institut für Quantenoptik, Hans-Kopfermann-Strasse 1, D-85748 Garching, Germany

E-mail address: ioachim.pupeza@mpq.mpg.de 